\title{
A NOTE ON THE CONVEXITY OF THE EXPECTED QUEUE LENGTH OF THE M/M/s QUEUE WITH RESPECT TO THE ARRIVAL RATE: A THIRD PROOF ${ }^{1}$
}

\author{
A. MEHREZ \\ lient Slate University \\ Graduate School of Management \\ Kent, Ohio 44020, U.S.A. \\ J. BRIMBERG \\ Royal Military College of Canada \\ Department of Engineering Management \\ Kingston, Ontario \\ CANADA K7K 5LO
}

\begin{abstract}
The convexity of the expected number in an $M / M / s$ queue with respect to the arrival rate (or traffic intensity) is well known. Grassmann [1] proves this result directly by making use of a bound on the probability that all servers are busy. Independently, Lee and Cohen [2] derive this result by showing that the Erlang delay formula is a convex function. In this note, we provide a third method of proof, which exploits the relationship between the Erlang delay formula and the Poisson probability distribution. Several interesting intermediate results are also obtained.
\end{abstract}

Key words: $M / M / s$ queue, arrival rate, expected queue length, convexity, Erlang delay formula.

AMS (MOS) subject classifications: $\quad 60 \mathrm{~K} 25$.

\section{INTRODUCTION}

Optimization of queueing systems is becoming an increasingly important topic in several applied areas, such as flexible manufacturing and telecommunications. In optimization, convexity is a desirable property, since a local minimum of a convex function is also a global minimum. Thus, standard descent methods may be used to obtain the optimal solution.

Pertaining to queueing systems, the convexity properties of various performance measures, such as average queue size and proportion of customers lost, have been studied with

\footnotetext{
${ }^{1}$ Received revised version: December, 1992.
} 
respect to the number of servers, service rate, traffic intensity and other variables. Certain results have justified the use of efficient marginal allocation algorithms to solve for the optimal allocation of limited resources. A partial list of relevant research includes Lee and Cohen [3], Yao and Shanthikumar [5] and Shanthikumar and Yao [4].

Here we prove that the average number of customers waiting in an $M / M / s$ queue is a convex function of the arrival rate (or traffic intensity). This result is well known, having been proven independently by Grassmann [1] and Lee and Cohen [2]. Grassmann's method uses a bound on the probability that all servers are busy to show that the second partial derivative is positive. Lee and Cohen derive the result by proving first, that the Erlang delay formula is a convex function. Their method uses induction to show that the numerator in the second derivative is a polynomial whose coefficients are all positive. The purpose of this paper is to highlight a completely different approach, which considers the functional relation between the mean queue size and the Poisson probabilities with parameter equal to the ratio of arrival rate to service rate. This permits a comparatively straightforward analysis of first and second order derivatives. Also, in developing the main result, several intermediate results are obtained which may be of interest.

\section{CONVEXITY OF THE EXPECTED QUEUE LENGTH}

All stochastic processes located in the paper will be considered on the probability space $(\Omega, \mathcal{F}, P)$.

Consider an $\mathrm{M} / \mathrm{M} / \mathrm{s}$ queue with arrival rate $\lambda$, service rate $\mu$ and $s$ servers. The traffic intensity is defined as usual by the ratio,

$$
\rho=\frac{\lambda}{s \mu}
$$

For equilibrium conditions to be attained, it is well known that $\rho$ must be less than one. Let us further define

$$
x=\lambda / \mu=s \rho .
$$

Then $x \in(0, s)$. The expected queue length is given by

$$
L_{q}=\frac{x C(s, x)}{(s-x)}
$$

where

$$
C(s, x)=\frac{x^{s}}{s !+(s-1) ! \sum_{k=1}^{s-1} \frac{(s-k) x^{k}}{k !}}
$$


is the Erlang delay formula. The expected number in the system,

$$
L=L_{q}+x \text {. }
$$

We now state the main result.

Theorem 1: $\quad L_{q}$ and $L$ are convex functions of $x$ in the interval $(0, s)$.

Proof: Since $L=L_{q}+x$, we only need to show that $L_{q}$ is convex with respect to $x$. Taking the first partial derivative, we obtain

$$
\frac{\partial L_{q}}{\partial x}=\frac{\left(C+x \frac{\partial C}{\partial x}\right)(s-x)+x C}{(s-x)^{2}} .
$$

Since $\partial C / \partial x>0$, it follows that $L_{q}$ and $L$ are monotonically increasing functions of $x$ in the interval $(0, s)$.

The second partial derivative is given by

$$
\frac{\partial^{2} L_{q}}{\partial x^{2}}=\frac{2 \frac{\partial C}{\partial x}+x \frac{\partial^{2} C}{\partial x^{2}}}{(s-x)}+\frac{2\left(C+x \frac{\partial C}{\partial x}\right)}{(s-x)^{2}}+\frac{2 x C}{(s-x)^{3}} .
$$

Thus, to show convexity it is sufficient to demonstrate that

$$
(s-x) \frac{\partial^{2} C}{\partial x^{2}}+2 \frac{\partial C}{\partial x} \geq 0 .
$$

To proceed, we first introduce the functions

$$
f_{h}(x)=\frac{e^{-x_{x} x^{h}}}{h !}, \quad h=0,1, \ldots
$$

Note that $f_{h}$ has the form of a Poisson probability with parameter $x$. The following recursive relations are obtained:

$$
\begin{aligned}
& f_{h}^{\prime}=f_{h-1}-f_{h}, \\
& f_{h}^{\prime \prime}=f_{h-2}-2 f_{h-1}+f_{h}, \\
& h=0,1, \ldots,
\end{aligned}
$$

where $f_{-2}=f_{-1}=0$, and the ' and "denote $\partial / \partial x$ and $\partial^{2} / \partial x^{2}$ respectively. The Erlang loss formula can be rewritten as a function of Poisson probabilities as follows:

We note some useful properties below:

$$
C(s, x)=\frac{s !}{(s-1) !} \frac{f_{s}}{\sum_{h=0}^{s-1}(s-h) f_{h}} .
$$

$$
\frac{\partial}{\partial x}\left[\sum_{h=0}^{s-1}(s-h) f_{h}\right]=-\sum_{h=0}^{s-1} f_{h}<0
$$




$$
\begin{gathered}
\frac{\partial^{2}}{\partial x^{2}}\left[\sum_{h=0}^{s-1}(s-h) f_{h}\right]=f_{s-1}>0 \\
\frac{\partial f_{s}}{\partial x}=e^{-x}\left[\frac{x^{s-1}(s-x)}{s !}\right]>0, \forall x \in(0, s) .
\end{gathered}
$$

After some straightforward steps, it is readily seen that proving relation $(A)$ is exactly equivalent to showing that the following inequality is true.

$$
\begin{gathered}
{\left[(s-x)\left(u^{\prime \prime} v-v^{\prime \prime} u\right)+2\left(u^{\prime} v-v^{\prime} u\right)\right] v} \\
-2 v^{\prime}\left(u^{\prime} v-v^{\prime} u\right)(s-x) \geq 0
\end{gathered}
$$

where $u=f_{s}$ and $v=\sum_{h=0}^{s-1}(s-h) f_{h}$.

The proof of relation $(B)$ consists of verifying the following components:

(i) $(s-x) u^{\prime \prime}+2 u^{\prime}>0$

(ii) $\left[-v^{\prime \prime} u v+2\left(v^{\prime}\right)^{2} u\right]>0$,

(iii) $-v^{\prime} u>0$,

(iv) $\quad-v^{\prime} u^{\prime}>0$.

Inequality (iii) follows immediately from property (1), while inequality (iv) follows by applying properties (1) and (3). Noting that $u^{\prime}=f_{s-1}-f_{s}$, and $u^{\prime \prime}=f_{s}-2 f_{s-1}+f_{s-2}$, we obtain

$$
(s-x) u^{\prime \prime}+2 u^{\prime}=\frac{f_{s}-2}{s(s-1)}(s-x)\left[(s-x)^{2}+2 x-s\right]
$$

for $s \geq 2$. (For $s=1$, the right-hand side becomes $x(1-x) f_{0}$, which is clearly $>0$.) Furthermore, the function, $(s-x)^{2}+2 x-s$, is convex in $x$, with minimum value of $(s-1)$ at $x^{*}=s-1$. We conclude that inequality $(i)$ is true.

Finally, to show that inequality (ii) holds, we must prove equivalently that

$$
f_{s-1} \sum_{h=0}^{s-1}(s-h) f_{h} \leq 2\left(\sum_{h=0}^{s-1} f_{h}\right)^{2}
$$

Using a change on index, the left-hand side may be rewritten as

$$
f_{s-1} \sum_{h=0}^{s-1}(s-h) f_{h}=e^{-2 x} \sum_{\ell=s-1}^{2(s-1)} \frac{(2 s-\ell-1) x^{\ell}}{(s-1) !(\ell-(s-1)) !}
$$

A lower bound for the right-hand side may be obtained by combining and deleting terms as follows:

$$
\left(\sum_{h=0}^{s-1} f_{h}\right)^{2}=e^{-2 x} \sum_{i=0}^{s-1} \sum_{h=0}^{s-1} \frac{x^{i+h}}{i ! h !}
$$




$$
>\epsilon^{-2 x} \sum_{\ell=s-1}^{2(s-1)}\left[\sum_{i=\ell-(s-1)}^{s-1} \frac{1}{i !(\ell-i) !}\right] x^{\ell}
$$

But clearly,

$$
\begin{aligned}
\sum_{i=\ell-(s-1)}^{s-1} \frac{1}{i !(\ell-i) !} & \geq(2 s-\ell-1) \quad \min _{\ell-(s-1) \leq i \leq s-1}\left[\frac{1}{i !(\ell-i) !}\right] \\
& =\frac{2 s-\ell-1}{(s-1) !(\ell-(s-1)) !}
\end{aligned}
$$

for $\ell=s-1, s, \ldots, 2(s-1)$. It immediately follows that relation $(C)$ is true.

We conclude that $L_{q}$ and $L$ are strictly convex functions of $x \in(0, s)$. Alternatively stated, $L_{q}$ and $L$ are strictly convex functions of the arrival rate $\lambda$ in the interval $(0, s \mu)$, or traffic intensity $\rho$ in the interval $(0,1)$.

\section{REFERENCES}

[1] Grassmann, W.K., The convexity of the mean queue size of the $M / M / c$ queue with respect to traffic intensity, J. of Appl. Prob. 20, (1983), 916-919.

[2] Lee, H.L. and (ohen, M.A., A note on the convexity of performance measures of M/M/c queueing systems, J. of Appl. Prob. 20, (1983), 920-923.

[3] Lee, H.L. and Cohen, M.A., Multi-agent customer allocation in a stochastic service system, Management Science 31, (1985), 752-763.

[4] Shanthikumar, J.G. and Yao, D.D., Optimal server allocation in a system of multiserver stations, Management Science 33, (1987), 1173-1180.

[5] Yao, D.D. and Shanthikumar, J.G., The optimal input rate to a system of manufacturing cells, Infor. 25, (1987), 57-65. 


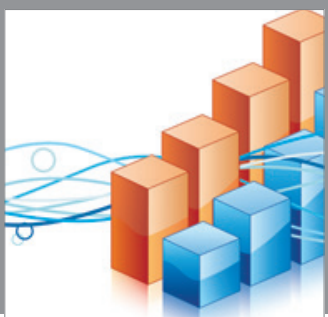

Advances in

Operations Research

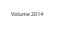

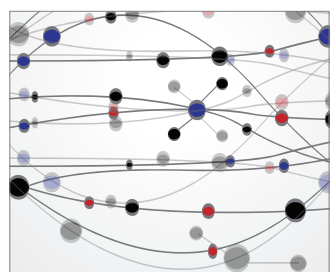

\section{The Scientific} World Journal
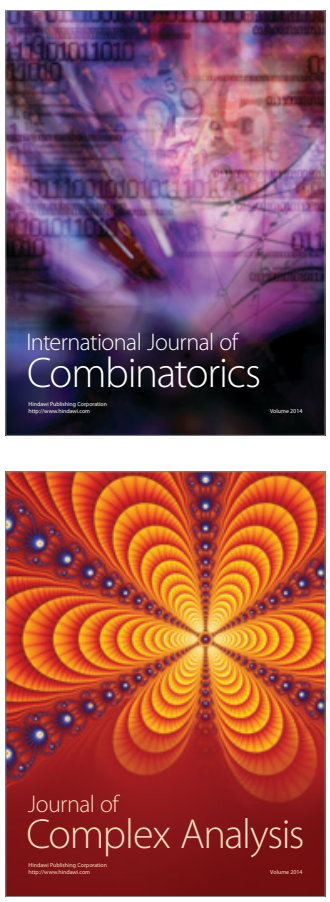

International Journal of

Mathematics and

Mathematical

Sciences
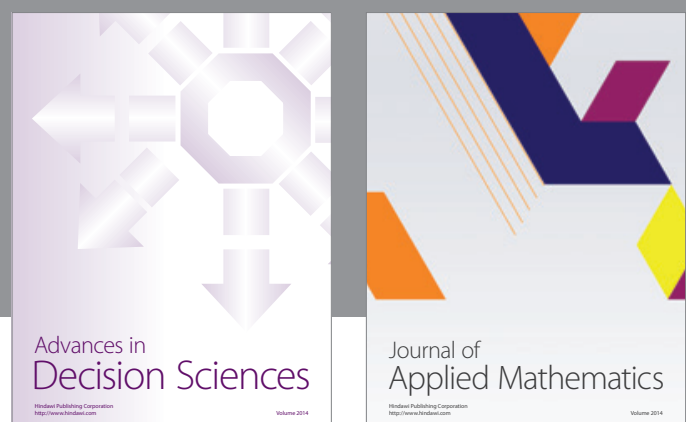

Journal of

Applied Mathematics
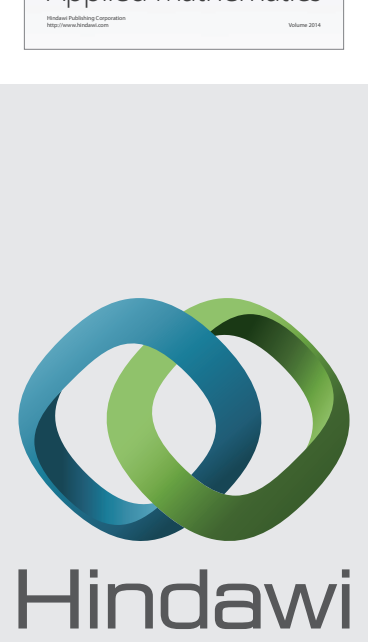

Submit your manuscripts at http://www.hindawi.com
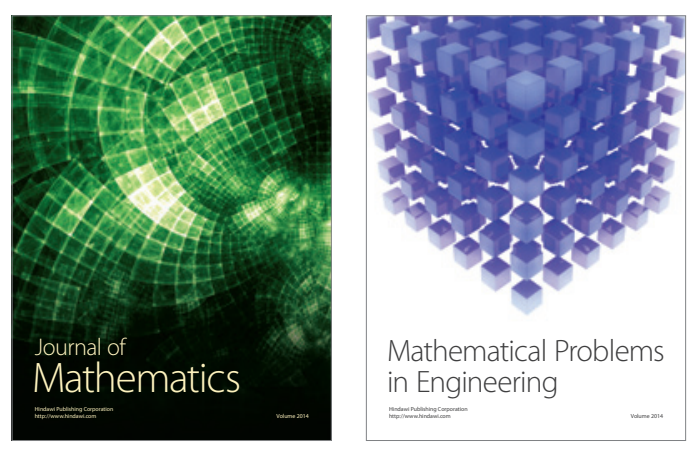

Mathematical Problems in Engineering
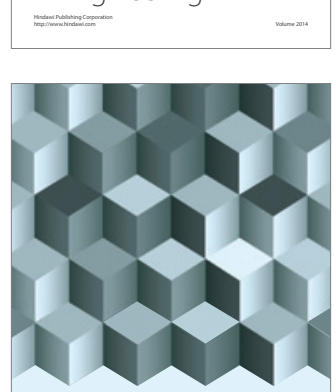

Journal of

Function Spaces
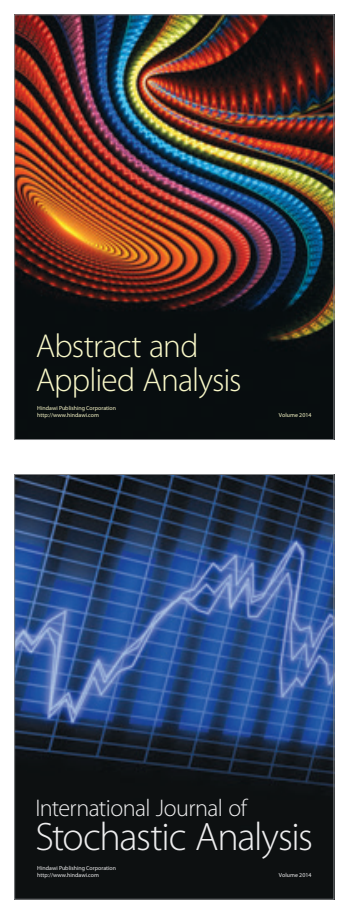

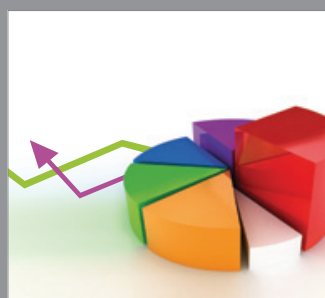

ournal of

Probability and Statistics

Promensencen
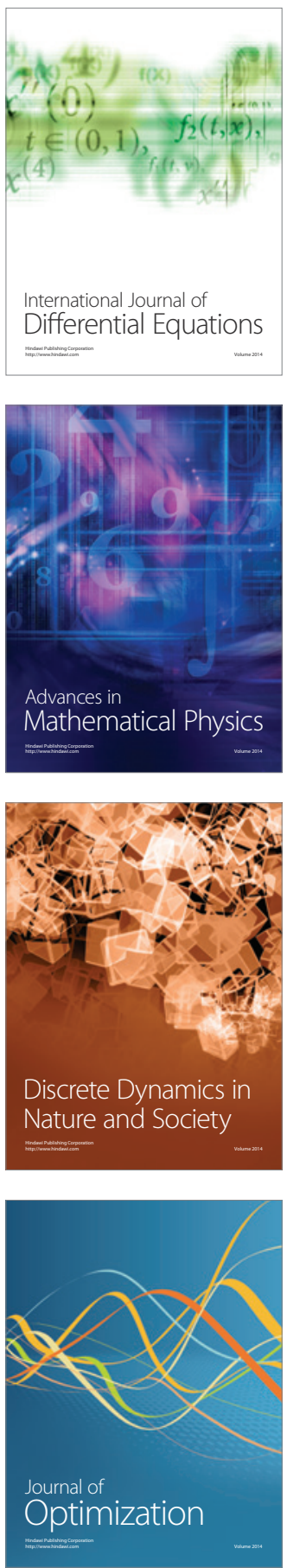